Revue de l'Institut des langues et cultures

d'Europe, Amérique, Afrique, Asie et Australie

37 | 2019

Des genres en Méditerranée : pratiques,

représentations et transfert

\title{
Appréhender la diversité langagière et culturelle au fil des textes et des discours : une entrée par la notion de « genre »
}

Approaching Linguistic and Cultural Diversity Through Texts and Discourses:

Entering Through the Notion of "text genre"

Monica Masperi, Triscia Biagiotti, Giada Quaranta et Martina Barletta

\section{OpenEdition}

Journals

Édition électronique

URL : http://journals.openedition.org/ilcea/7430

DOI : 10.4000/ilcea.7430

ISSN : 2101-0609

Éditeur

UGA Éditions/Université Grenoble Alpes

Édition imprimée

ISBN : 978-2-37747-099-0

ISSN : $1639-6073$

Référence électronique

Monica Masperi, Triscia Biagiotti, Giada Quaranta et Martina Barletta, « Appréhender la diversité langagière et culturelle au fil des textes et des discours : une entrée par la notion de « genre » », ILCEA [En ligne], 37 | 2019, mis en ligne le 04 novembre 2019, consulté le 15 janvier 2020. URL : http:// journals.openedition.org/ilcea/7430 ; DOI : 10.4000/ilcea.7430

Ce document a été généré automatiquement le 15 janvier 2020.

(c) ILCEA 


\section{Appréhender la diversité langagière et culturelle au fil des textes et des discours : une entrée par la notion de « genre»}

Approaching Linguistic and Cultural Diversity Through Texts and Discourses:

Entering Through the Notion of "text genre"

Monica Masperi, Triscia Biagiotti, Giada Quaranta et Martina Barletta

\section{Introduction}

1 La présente contribution s'inscrit dans le cadre d'une recherche en didactique initiée au sein de l'IDEFI Innovalangues (Masperi, 2011) ${ }^{1}$, portant sur la conception de parcours numériques de formation en langues. Ces réalisations didactiques s'élaborent autour de séquences vidéo sélectionnées sur la base de valeurs culturelles qui les désignent comme étant représentatives de la langue-culture cible. Dans ce contexte, la notion de "genre de texte» constitue le pivot autour duquel s'échafaude une approche didactique fortement adossée à la recherche fondamentale (en L1, mais quasiment inexplorée en $\mathrm{L} 2)^{2}$ et qui place la langue orale au centre de ses préoccupations.

2 Nous allons ici questionner d'une part ce parti pris didactique qui se positionne, sous certains aspects, à contre-courant des pratiques formatives en L2 des dernières décennies. D'autre part, nous interrogerons cet objet difficile d'enseignementapprentissage qu'est l'« oral », dont la complexité apparaît d'autant plus manifeste que l'on tente, comme nous le proposons, de l'appréhender sans la médiation de l'écrit. 


\section{Aborder les textes par le "genre » : repères théoriques}

3 Du point de vue didactique, le texte est traditionnellement considéré comme l'unité de base de l'enseignement tant de la production que de la réception (orale et écrite). Lorsqu'on se représente une entrée dans les textes en L2 via un environnement numérique s'inscrivant dans des pratiques formatives institutionnalisées, ce n'est pas à proprement parler la conceptualisation partagée du texte en tant qu'objet complexe - solidement articulé, culturellement dense et socialement ancré - qui fait surface. Les approches communicatives et les démarches actionnelles, fortement ancrées au CECRL, ont habitué enseignants et concepteurs à orienter la sélection de textes à partir de contenus considérés comme prioritaires pour l'accès à la langue-culture cible et sur la base de matériaux langagiers permettant de confectionner des outils didactiques (Plane \& Schneuwly, 2000) et de concevoir des enchainements d'activités selon une logique de progression graduée, fidèle aux descripteurs.

4 Quelles innovations éducatives et sociales pourraient être accueillies (Masperi \& Quintin, 2014) dans ce paysage de modèles largement admis, de pratiques ritualisées, déléguant pour l'essentiel à la modalité de l'interaction et de la médiation formative - distancielle ou hybride, médiatisée par un environnement numérique - toute initiative de transformation des pratiques didactiques?

5 Au-delà de la conception de la charpente du parcours numérique de formation, du format du dispositif et des modalités d'accompagnement qui peuvent y être associées, que pouvons-nous proposer d'innovant lorsqu'on s'intéresse à une approche didactique qui prend appui sur la notion de texte inscrit dans des pratiques langagières socialement partagées et présenté en tant que résultat observable de cette activité (Bronckart, 2008) ?

6 De quelle manière aborder ce texte en profondeur, donner à voir les formes et les contenus de la langue-culture qu'il véhicule, ainsi que leur mode d'articulation, et ceci de manière " dématérialisée ", en mettant à profit les potentialités d'un environnement numérique de formation?

7 C'est du côté d'une réflexion menée de longue date en français langue première en Suisse romande que nous sommes allées chercher dans un premier temps des éléments de réponse à nos questions. En l'occurrence, il nous a semblé particulièrement pertinent de nous appuyer sur le modèle de l'activité langagière, tel qu'il est exploré et décrit par J.-P. Bronckart $(1996,1997,2004,2008)$ car il présente l'intérêt d'ancrer l'activité langagière dans le fonctionnement social de la langue-culture à travers la notion de "genre». Définir la notion de "genre» n'est pas chose simple ${ }^{3}$. Pour la cerner, il est d'usage de considérer l'ancrage social, la nature discursive et les caractéristiques formelles de la production langagière. Sous cet angle, le «genre » peut se définir comme un ensemble de productions langagières - orales ou écrites - qui dans une culture donnée possèdent des caractéristiques partagées, de différents ordres (communicatif, pragmatique, sémantique, grammatical, graphique. Schneuwly, 1995 : 78). Ces « ensembles ", dont les formes tiennent à une série de choix fixés par l'usage, sont en principe souples, donc prédisposés à la mutation (Bakhtine, 1984; Adam, 1992, 1997 ; Bronckart, 1996) : ils peuvent naître, mourir, évoluer dans le temps, se détourner des motivations qui les ont engendrés pour se rendre autonomes et disponibles pour 
servir d'autres finalités, dans d'autres contextes. Cette variation potentielle, tributaire de l'échelle du temps et du contexte historico-culturel dans lequel les "genres" s'inscrivent, n'empêche toutefois pas ces entités de rester relativement stables dans le temps et de partager des faisceaux de traits communs qui concourent précisément à en tracer les contours et à leur attribuer un caractère distinctif. Tout texte - en tant que produit d'échanges socialement construits dans un contexte socio-historique donné relève donc d'un "genre ", de manière plus ou moins tranchée. C'est dans ce "moule englobant » que le texte prend corps et se façonne. Et si tout texte est « singulier », son schéma formel, s'actualisant dans une pratique, une époque, une culture, inscrit ce texte "singulier » dans un format collectif, dans une « classe » de textes. Tout membre d'une communauté est donc confronté à un univers de textes "déjà là » (Bronckart, 1996), organisé en " genres » empiriques et historiques. Socialement et culturellement, tout texte est donc un exemplaire de genre (Dolz \& Gagnon, $2008: 181$ ).

Partant, nous postulons que n'importe quel locuteur qui s'engage dans une action de compréhension ou de production d'un texte, seul ou en interaction avec d'autres locuteurs (présents ou absents), dispose d'une connaissance des genres que sa culture lui a transmise et qui se concrétise dans l'activité langagière. De ce fait, ces formats langagiers que sont les genres textuels peuvent se révéler particulièrement pertinents en didactique.

Par ailleurs, ce modèle de l'activité langagière nous semble également orienter efficacement la compréhension de la langue en favorisant la perception de la valeur de ses unités à travers un format discursif socialement partagé. C'est la notion de « type de discours » qui sera alors convoquée pour délimiter dans un texte les séquentialités (cf. Adam, 1987, 1997, 2015) qui présentent une organisation particulière des données de la langue et pour identifier de quelle manière ce texte met en œuvre et donne à voir l'énonciateur, le destinataire et la finalité poursuivie à travers le mode de traitement du contenu.

Ce modèle de référence ${ }^{4}$ semble donc offrir au didacticien de langue un solide développement des propositions du CECRL en matière à la fois de texte, de genre et de discours.

\section{Aborder le texte par son " genre » : une posture émergeante en L2?}

11 Nous savons que tout enseignement de la langue implique la segmentation de la matière à enseigner et sa répartition sur l'axe du temps. Cette "matière " - l'activité langagière, en réception et en production - permet à l'homme de participer, communiquer, agir dans le monde. Elle est caractérisée par une très grande élasticité en cela qu'elle est en mesure de se diversifier et de se spécialiser en interaction permanente avec l'activité humaine et sa complexité. L'offre de formation en langue "par niveau », longitudinale et extensive, telle qu'elle est préconisée et pratiquée le plus souvent au niveau institutionnel, est censée catégoriser et distribuer cette matière sur une temporalité longue et la modéliser en fonction des objectifs établis par les filières (langue à usage général vs langue à usage professionnel). En dehors de formations hautement spécialisées, la progression établie pour la langue "de service " permet rarement de se donner le temps de saisir les textes dans leur complexité. Le caractère fugace et morcelé des productions auxquelles nous sommes constamment 
exposés via les réseaux sociaux contraste avec la posture qu'il est nécessaire d'adopter pour composer avec des discours fortement articulés et chargés culturellement. Qui plus est, lorsqu'on se situe sur le plan de l'oralité notamment, il est de coutume de pointer la «pauvreté de l'enseignement de l'oral » (De Pietro, Gagnon \& Fisher, 2017), que ce soit en compréhension ou en production, autant en L1 qu'en L2. Habileté des plus complexes, la compréhension de l'oral en L2 est une activité langagière qui demeure mal connue (Catoire, 2017) : si apprenants et enseignants s'accordent à dire que les obstacles sont nombreux, ceux-ci sont souvent mal identifiés. Et pour cause : le regard didactique porte pour l'essentiel sur le résultat de la compréhension et beaucoup plus rarement sur les difficultés qui surgissent lors des processus de perception et d'accès au sens.

12 Mais préalablement à tout dessein de modélisation didactique, peut-on poser le « genre » comme objet d'apprentissage à part entière ? Peut-on le poser comme objet d'apprentissage de la compréhension de l'oral? Que pourrait apporter, de plus ou de différent, une approche des textes - et des textes oraux en particulier - par les "genres"? De quelle manière cette approche pourrait-elle contribuer à favoriser les acquisitions langagières en L2 ?

Pour avancer dans l'exploration de ces questionnements, nous avons commencé par extraire les concepts théoriques de leur réseau épistémologique d'origine afin de les reconfigurer dans une perspective didactique. À la base de la démarche, une idée mobilisatrice : la conviction que cette reconfiguration didactique nous permettrait de repenser ce qu'il y a d'universel dans le fonctionnement du langage et de ce fait de favoriser la transversalité dans l'approche de textes issus de différentes traditions culturelles. En effet, en dépit de l'empreinte idiosyncrasique propre à chaque langueculture et du constat que certains genres diffèrent sensiblement d'une culture à l'autre, tout texte pourra être rapproché à d'autres textes produits dans des situations langagières et des contextes socio-historiques similaires et donc reconduit à un «ensemble " qui permettra son repérage en tant qu'exemplaire d'un "genre ». Capitaliser sur ces connaissances, que nous pensons plutôt implicites - ou épilangagières (Gombert, 1990) - mais partagées, transversales, disponibles, permettrait à l'apprenant qui pratique et intériorise la reconnaissance de ces «enveloppes" que sont les genres, d'accroître ses capacités langagières (Dolz \& Gagnon, ibid.).

Pour jeter les bases de notre construction didactique, nous avons ainsi fait le choix de nous appuyer sur le modèle de l'activité langagière de Bronckart et de le mettre en relation dialogique avec les travaux d'autres théoriciens du langage (comme J-M. Adam, F. Rastier, M. Burger) et de didacticiens du français langue première ou seconde qui s'inscrivent dans ce sillage épistémologique, tels que B. Schneuwly, J. Dolz, R. Gagnon (Dolz \& Schneuwly, 1998 ; Dolz \& Gagnon, 2008) et S. Chartrand (2015, 2016). Les travaux de Chartrand notamment sont particulièrement inspirants dans la perspective d'un transfert de la L1 vers la L2. 


\section{Entrer par le « genre » dans la compréhension de l'oral : nos fondamentaux en L2}

15 Toute production verbale, orale ou écrite, mobilise des unités de taille variable (du morphème à l'enchâssement de phrases) qui s'articulent entre elles de manière plus ou moins conventionnalisée pour former des textes. Unité attestable de l'activité langagière et unité fonctionnelle de la communication (Dolz \& Ganon, 2008: 186), le texte, nous l'avons évoqué, se pose comme le noyau de base de l'enseignement. La centration sur cet objet pose au didacticien à la fois des questions de sélection et de classement. Aux choix qui s'imposent en termes de recueil de ressources textuelles mobilisables, conditionnés à la fois par l'hétérogénéité des productions et par l'utilisation de ces supports (et des contraintes liées aux droits d'exploitation) s'ajoute la difficulté de classement de ces textes. Le classement des textes par «genre » ou par "types de discours » reste dans les usages en L2 assez marginal, ce qui est sans doute révélateur de la faiblesse de l'outillage didactique préposé à ce type de catégorisation, mais également d'un certain manque de familiarité avec les concepts qui le soustendent. Savoir identifier un texte comme appartenant à un genre et déceler à l'intérieur de ce couplage un type de discours est en effet une tâche complexe : cela implique de saisir et de mettre dans une relation de co-textualité particulière l'ensemble des unités linguistiques que le texte nous offre d'une part et d'autre part à dégager de ces mêmes unités les formes discursives spécifiques de sa textualité (Dolz \& Ganon, ibid. : 182). En prenant la mesure de ces éléments de difficulté, nous avons posé nos choix en matière de construction didactique de parcours numériques sur la base de deux principes cardinaux : définir et orienter rigoureusement la sélection des contenus langagiers et définir les procédures susceptibles de faciliter l'accès à ces contenus. Ces procédures, configurées dans un premier temps pour faciliter la compréhension et la production des textes seront ensuite reconfigurées pour être intégrées dans l'environnement numérique (Biagiotti \& Quaranta, à paraître).

\subsection{Sélection des contenus}

16 Chaque unité didactique $(\mathrm{N}=4)$ de nos parcours $(\mathrm{N}=6)$ est construite à partir d'un texte qui relève d'un genre de texte et d'un type de discours donné. Les contenus proposés sont tirés de ressources vidéos originales ${ }^{5}$, sélectionnées sur la base de leur "densité culturelle ». Dans une perspective socio-discursive, il nous semble en effet essentiel de disposer de vidéos représentatives des valeurs socioculturelles de la langue et de la culture cible et clairement inscrites dans une pratique sociale largement partagée par les parlants d'une communauté donnée. Deux autres critères de sélection sont pris en considération :

a. le texte doit s'inscrire dans une pratique sociale facilement identifiable et donc relativement stabilisée du point de vue culturel. Nous considérons ce critère comme étant un élément facilitateur dans l'identification du genre.

b. le texte doit donner clairement à voir l'organisation spécifique de ses unités (à savoir, le «type de discours »). La redondance d'un même type de discours à l'intérieur du parcours a pour effet de créer un lien entre des textes qui relèvent de genres différents, ce qui renforce la cohésion du parcours. 
17 Le texte doit enfin être extrait de son contexte d'origine pour devenir un «objet d'enseignement »: les composantes visuelles et langagières seront repensées en fonction de cet objectif, en prêtant le plus grand soin à la sélection des extraits à didactiser.

\subsection{Procédures didactiques}

Pour exploiter ces ressources d'un point de vue didactique, nous avons adopté une approche des données de langue qui part de la globalité du texte et s'intéresse ensuite à ses constituants, adoptant un grain d'analyse de plus en plus fin: du "genre», au «type de discours" jusqu'au travail sur les unités de la langue, à savoir lexique, morphosyntaxe, phonétique et prosodie.

\section{Genres de texte}

19 Médiateurs de l'activité humaine, les genres orientent la réalisation de toute action langagière (Dolz \& Gagnon, 2008). Ils sont nommés, identifiés et catégorisés par les usages (ibid.: 187). De ce fait, nous l'avons souligné (cf. supra, 2), chaque locuteur possède une représentation plus ou moins étendue des genres disponibles dans sa langue-culture. Notre objectif est d'activer d'emblée chez l'étudiant, placé face au texte, sa capacité à tirer parti de la connaissance sur les genres dont il dispose dans sa langueculture et dans celle qu'il est en passe de se construire dans la langue d'apprentissage. Pour ce faire, l'apprenant sera invité à s'interroger sur le genre de la ressource qui lui est proposée : il sera pour cela sollicité à identifier, en première approche, les indices de type contextuel que le texte lui offre. Cette identification concourt à forger la représentation du genre de texte auquel il est exposé.

\section{Types de discours}

Les types de discours, nous l'avons évoqué, sont des formes de planification et d'organisation conventionnelles du contenu, caractérisées par des procédés linguistiques spécifiques, par une organisation particulière des unités de la langue. Ils constituent ainsi des «modèles » disponibles dans chaque langue-culture. La notion de "type de discours", à côté de celle de "genre de texte ", s'avère centrale pour la construction de capacités langagières des apprenants. Le préalable à cette construction nous semble cependant être la conscience que l'apprenant doit acquérir de ces modèles, de leurs traits spécifiques, de leur organisation. Cette conscience, nous en faisons l'hypothèse, permettrait de faciliter non seulement la compréhension mais également la production de textes empiriques qui s'inscrivent dans des genres contextualisés, dans les pratiques sociales de la langue-culture, objet d'apprentissage.

Le travail de regroupement facilitant le déploiement des contenus de formation, c'est l'entrée par «types de discours » que nous avons privilégiée afin d'organiser le contenu de notre formation en une série de "parcours". Ceux-ci relèvent de la typologie couramment admise dans la littérature (Adam, 1987 et suiv., cf. supra), à savoir : informatif, explicatif, narratif, argumentatif, injonctif, poétique. Dans le processus de reconfiguration didactique ces étiquettes académiques ont été retravaillées pour aboutir à: informer, expliquer, raconter, argumenter, faire agir et "jouer avec la langue ». Cette dernière catégorie, très ouverte, regroupe les "textes de création». Chaque 
parcours se compose ainsi de textes qui renvoient à des genres différents, mais qui se caractérisent par le même type de discours dominant.

\section{Entrer par l'« oral » dans la compréhension de l'oral : nos lignes-forces en L2}

"Ça va très vite ", «c'est tout lié », « ils parlent avec un accent », « je ne comprends pas la moitié des mots...»: la compréhension de l'oral est une activité langagière essentielle, extrêmement mobilisatrice sur le plan cognitif, intimidante. Beaucoup d'encre a coulé à son sujet (e.g. Field, 2008, 2019; Cornaire, 1998; Hilton, 2000 ; Bloomfield et al., 2010 ; Catoire, 20017 ; De Pietro et al., 2017) et nombre de questions restent ouvertes (De Pietro et al., ibid. : 11) quant à la définition et à la délimitation de cet objet, aux objectifs à atteindre, à la progression à planifier tant en L1 qu'en L2. Le peu d'entraînement à la perception et à l'écoute active, sans la médiation de l'écrit, pratiqué en situation d'apprentissage formel en France, couplé au conditionnement perceptif induit chez l'auditeur par les propriétés sonores du français ${ }^{6}$ (seule «langue première » du répertoire langagier de la plupart de nos publics), fait que, le plus souvent, l'apprenant francophone n'est pas en capacité d'extraire et de traiter avec aisance la matière phonique d'un flux sonore continu en L2 articulé de manière naturelle (Bosworth, 2001). Sur le plan suprasegmental, l'attention portée aux caractéristiques accentuelles et rythmiques de la langue cible et par là à des phénomènes fondamentaux en termes de compréhension (i.e. segmentation, phénomènes d'enchaînement et de restructuration syllabique) ou encore à la prise en compte des indices prosodiques en tant que moteurs de processus d'inférence, est pour ainsi dire absente des pratiques de classe (Baqué et al., 2003 ; Picavet et al., 2013).

Lorsque nous avons posé le cadre conceptuel de notre approche, nous avons pris le parti, assez radical, de traiter l'oral sans la médiation de l'écrit. A cet effet, nous avons conçu une série d'outils techno-pédagogiques qui permettent à l'étudiant de manipuler le document sonore au fil du texte (Koszul \& Petitgirard, 2014), de manière à faciliter le processus de traitement de l'oral, in itinere, au moment de l'écoute. L'idée est donc de renverser la tendance ancrée dans les pratiques formatives (numériques, notamment), qui consiste à évaluer ex post le résultat du traitement du texte (qu'avez-vous compris ?), pour soutenir et accompagner les processus auditifs et les stratégies d'écoute et de compréhension (qu'êtes-vous en train d'entendre et de comprendre ?).

Nous allons ici proposer un bref aperçu de deux de ces outils d'assistance à la compréhension : le «Bornage Audio » et le « Chapitrage Audio et Vidéo ».

\section{« Bornage Audio »}

Le Bornage Audio est un protocole d'activité qui demande à l'apprenant d'identifier un extrait audio par la sélection d'une zone à l'intérieur de la représentation visuelle de la séquence sonore (i.e. la waveform ou forme d'onde associée à une ligne de temps). Le concepteur détermine la ou les zones de réponse correctes ou incorrectes et fixe une tolérance de dépassement de zone à droite et à gauche, correspondant au début et à la fin du segment. Il ajoute également le feedback à afficher. L'étudiant indique sa ou ses réponse(s), grâce à des taquets qui «bornent » une ou plusieurs zones sur la forme d'onde. Les zones sélectionnées par l'étudiant sont analysées et comparées à celles 
déterminées par le concepteur en tenant compte de la tolérance indiquée dans les paramètres de l'activité. Un feedback est affiché en conséquence.

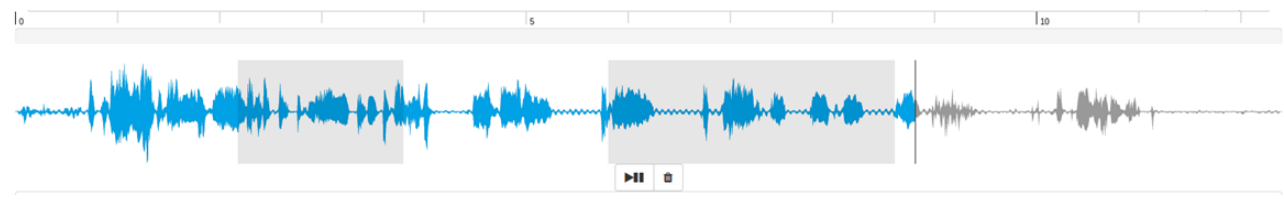

Figure 1 : Bornage Audio.

Ce qui peut être donné à repérer c'est un élément saillant du texte sur lequel porte l'effort de compréhension, des éléments significatifs au niveau phonique typique de la langue orale (enchaînements et resyllabifications, courbes expressives, variétés diatopiques...) ou encore tout élément pertinent d'ordre lexical, morphologique ou syntaxique.

\section{Outil « Chapitrage Audio et Vidéo $»^{7}$}

Le chapitrage est un « Enriched Media Object » (EMO) ${ }^{8}$ conçu pour écouter ou visionner un extrait audio ou vidéo de manière segmentée, permettant d'isoler et de mettre en évidence des éléments présents dans l'extrait sonore ou visuel. L'étudiant peut revenir sur un segment autant de fois que nécessaire et l'écouter avec un débit variable. À chaque segment peuvent être associés un titre, un sous-titre et une note explicative. Le chapitrage n'est pas un protocole d'activité, en ce sens qu'il ne gère pas une interaction pédagogique de type question/réponse. Il doit donc être associé à un protocole d'activité spécifique (QRM, QRU, association, classement, ordonnancement, tableau...) en mesure de fournir un feed-back.

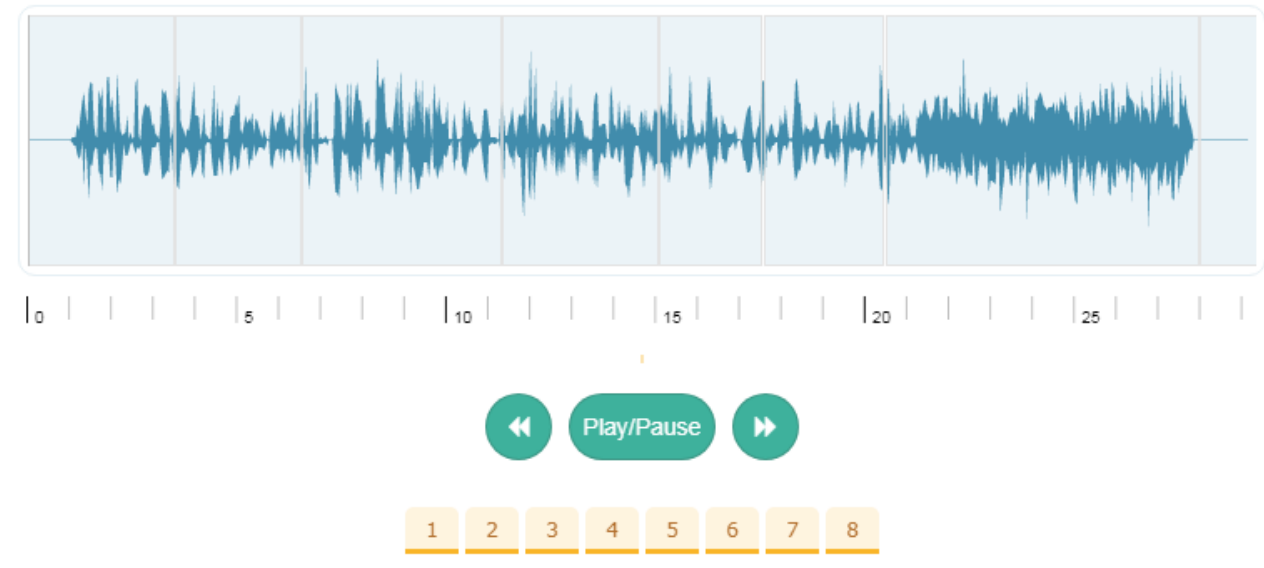

Figure 2 : Chapitrage Audio.

\subsection{Illustration d'une unité prototypique (italien L2)}

Des exemples d'activités tirées d'unité du parcours «Expliquer - en route vers B2 » en italien nous aideront à illustrer de quelle manière les procédures didactiques envisagées ont été concrètement mises en œuvre.

L'unité s'échafaude autour d'une conférence "grand public » tenue par un critique d'art très médiatisé en Italie - Vittorio Sgarbi - et portant sur une figure emblématique et controversée de la peinture italienne du XVII ${ }^{\mathrm{e}}$ siècle : Caravage. 

indices sur le genre et sur le type de discours dominant : le nom du conférencier, le titre de la conférence, l'indication du lieu où la conférence se tient et de la manifestation culturelle qui l'accueille. L'entrée dans le texte se fait par un court extrait vidéo servant de support à une activité appelée " ancrage dans le genre ». Le visionnage de l'extrait est précédé d'une consigne sonore explicitant en langue cible la capacité que l'activité mobilise $^{9}$ : «identificare il genere » (identifier le genre). À l'occasion de cette première activité, l'étudiant est invité à formuler des hypothèses sur le genre du texte. L'émergence de ces hypothèses est suscitée par une série de propositions (sonores). Les étudiants devront sélectionner les options qu'ils considèrent comme étant les plus proches de leurs propres intuitions. A priori, rien ne s'oppose à ce que toutes les options proposées soient plausibles. L'occasion sera fournie à l'étudiant de vérifier le bien-fondé de ces hypothèses tout au long de l'unité, grâce à la découverte progressive des extraits vidéos auxquels il sera exposé et aux activités qui lui seront proposées ${ }^{10}$.

La découverte du texte se poursuit par une invitation au questionnement sur les caractéristiques discursives de la vidéo. La capacité mobilisée est explicitement donnée à entendre par la consigne: "identificare il tipo di discorso » (identifier le type de discours). À partir de là, une série d'activités de réflexion et de sensibilisation aux types de discours est proposée. La dimension discursive est appréhendée à un niveau plus fin de granularité : la « capacité » à mobiliser dans ce type d'activités est précisée ${ }^{11}$ et les protocoles d'activités privilégient un traitement de la bande sonore ciblé sur l'exploration et l'extraction d'un contenu spécifique. s'agit d'identifier un élément explicatif spécifique (la raison pour laquelle Caravage serait assimilé à un peintre du XIX ${ }^{e}$ siècle). L'EMO «Chapitrage » est ici associé à un protocole de type « question à réponse unique » $(\mathrm{QRU})^{12}$.

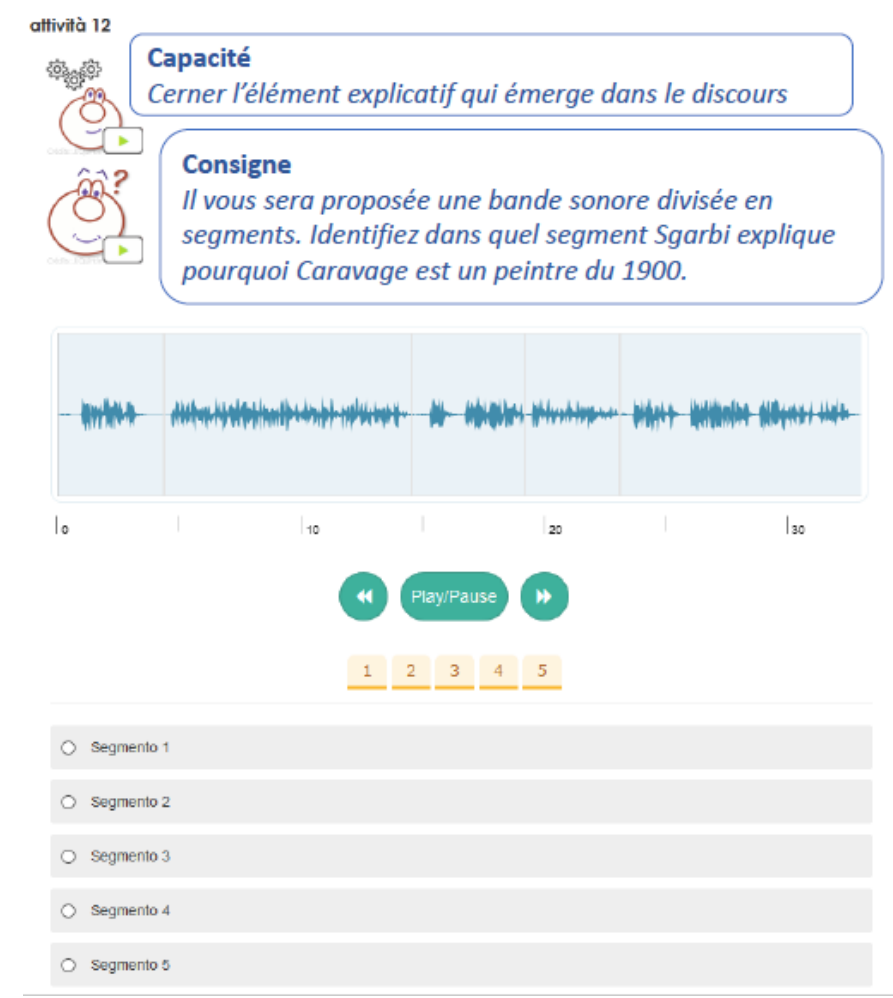

Figure 3 : Chapitrage Audio avec protocole QRU. 


\section{En guise de conclusion prospective}

L'approche par les "genres » que nous avons présentée apporte un éclairage nouveau sur l'objet-texte, conduisant l'apprenant à se représenter différemment l'appréhension de cet objet et l'enseignant à modifier sa représentation de la réception et de la production textuelle, bousculant une certaine tradition dans la formation en langues. Les procédures adoptées tendent à sensibiliser nos publics à des traits universels du fonctionnement langagier et à la manière dont chaque langue les fait vivre dans les usages. Cette prise de conscience devrait aider les apprenants à développer et à mettre en interaction des capacités langagières complexes, notamment en réception. L'entrée dans le texte que nous proposons, plaçant explicitement en arrière-plan les questions de contenus pour faire ressortir et traiter en profondeur les éléments de type contextuel, structurel et discursif, devrait permettre de soutenir la persistance dans l'effort de saisie de textes complexes, à forte valeur culturelle, et d'accroitre la confiance dans la manière de les aborder sur le plan langagier. Le choix du traitement exclusif par l'oral de la dimension sonore et visuelle du texte, soutenu par un outillage techno-pédagogique d'assistance à la perception et à la compréhension, devrait mettre l'étudiant en condition de gagner progressivement en aisance dans ce type d'activité langagière. Si la voie empruntée semble prometteuse en termes d'effet transformant sur les pratiques formatives, la " preuve du concept » reste à faire. La stabilisation de la modélisation didactique a nécessité en effet un temps long de maturation, si bien que les premiers pilotages sur le terrain sont récents et les procédures d'évaluation (questionnaires et protocoles think aloud) encore instables. Ces premiers coups de sonde nous livrent quelques « impressions » certes encourageantes, mais qui ne peuvent faire figure de résultat de recherche. Un plan expérimental sera appliqué à partir de la rentrée 2019, dont l'ambition est d'asseoir nos principes méthodologiques, de transposer la démarche vers d'autres langues et in fine de traduire ces "démonstrateurs » en un produit éditorial diffusable à plus large échelle. Cet objectif s'accompagne d'une autre perspective de travail, qui entend faire émerger les relations qui existent entre le texte travaillé et d'autres textes ancrés dans la même pratique sociale. Pour ce faire, et toujours dans le but de cibler la langue de culture, nous envisageons d'intégrer dans chaque parcours didactisé un corpus de vidéos triées et indexées. Les usagers (enseignants, chercheurs, étudiants) pourront les visionner pour développer leur capacité à identifier les pratiques sociales, les genres et les types de discours dans une situation plus ouverte que celle d'un travail guidé sur un texte didactisé, faisant figure d'illustration simplifiée et aménagée, notamment par le choix didactique de délimiter les segments sur lesquels centrer l'analyse. Cet ensemble d'outils, intégré au sein d'un dispositif ouvert et évolutif, devrait, nous l'espérons, contribuer à développer le goût d'une approche approfondie des textes en langueculture cible et accroitre la prise d'autonomie dans l'apprentissage, tant sur le plan méthodologique que sur le plan langagier. 


\section{BIBLIOGRAPHIE}

ADAM Jean-Michel (1987), « Textualité et séquentialité. L'exemple de la description », Langue française, 74 (1), 51-72.

ADAM Jean-Michel (1992), Les textes types et prototypes. Récit, description, argumentation, explication et dialogue, Paris : Armand Colin.

ADAM Jean-Michel (1997), « Genres, textes, discours: pour une reconception linguistique du concept de genre », Revue Belge de Philologie et d'Histoire, 3(75), 665-681.

ADAM Jean-Michel (2015), Faire texte. Frontières textuelles et opérations de textualisation, Besançon : Presses universitaires de Franche-Comté.

BAKHTINE Mikhaïl (1984), Esthétique de la création verbale, Paris : Gallimard.

BAQUÉ Lorraine, LE BESNERAIS Martine \& MASPERI Monica (2003), « Entraînement à la compréhension orale des langues romanes. Quelques repères pour faciliter la prise en compte de la matière phonique ", Lidil - Revue de linguistique et de didactique des langues, 28, 137-152), <https:// journals.openedition.org/lidil/1853> (28 février 2019).

BIAGIOTTI Triscia \& QUARANTA Giada (à paraître), « Approcher les textes par les notions de " genres » et de « types de discours » : présupposés didactiques et mise en œuvre au sein d'un environnement numérique », Actes du colloque CEDIL (Colloque International des Étudiants chercheurs en Didactique des Langues et Linguistique), UGA, Grenoble.

BloomfieLD Amber, WAYLAND Sarah C., RHOADES Elizabeth, BlodgetT Allison, LinCK Jared \& Ross Steven (2010), « What makes listening difficult? Factors affecting second language listening comprehension », en ligne sur : <https://www.researchgate.net/publication/277788621> (27 février 2019).

BOsWORTH Gerôme S. (2001), « Mission Impossible ? Understanding English with French Ears », Le journal de TESOL France, 8, 1-12.

BRONCKART Jean-Paul (1996), Activité langagière, textes et discours : pour un interactionisme sociodiscursif, Lausanne : Delachaux et Niestlé.

BRONCKART Jean-Paul (1997), « La temporalité des discours, comme contribution à la reconfiguration des actions humaines », Travaux du centre de recherches sémiologiques, 65, 39-65.

BRONCKART Jean-Paul (2004), « Les genres de textes et leur contribution au développement psychologique ", Langages, 38(153), 98-108.

BRONCKART Jean-Paul (2008a), « Du texte à la langue, et retour. Notes pour une « reconfiguration » de la didactique du français ", Pratiques, 137/138, 97-116.

BRONCKART Jean-Paul (2008b), « Genres de textes, types de discours, et « degrés » de langue », C. Gerard (dir.), Texto, XIII, 1/2, en ligne sur le site : <http://www.revue-texto.net/index.php? $\mathrm{id}=86>$ (27 février 2019).

BURGER Marcel [dir.] (2010), « Langues et littérature pour l'enseignement du Français en Suisse romande : problèmes et perspectives ", Cahiers de L'Institut de linguistique et des sciences du langage, 27, <https://www.unil.ch/clsl/files/live/sites/clsl/files/shared/CILSL27.pdf> (28 février 2019). 
BURGER Marcel \& JACQUIN Jérôme (2015), « La textualisation de l'oral : éléments pour une description de la construction collaborative de la complétude », J.-M. Adam (dir.), Faire texte, Besançon : Presses Universitaires de Franche-Comté, 277-317.

CANELAS-TREVISI Sandra (2009a), La grammaire enseignée en classe. Le sens des objets et des manipulations, Berne : Peter Lang.

CANELAS-TREVISI Sandra \& BAIN Daniel (2009b), « La grammaire scolaire au service de l'enseignement-apprentissage du texte argumentatif ? Analyse critique de quelques pratiques en classe ", J. Dolz \& C. Simard (dir.), Pratiques d'enseignement grammatical, Québec : Les presses de l’Université Laval, 155-176.

CANELAS-TREVISI Sandra (2015), « Les subordonnées relatives et les textes en contexte : une rencontre impossible ?», P. Trevisiol \& M. Kaheraoui (dir.), Les subordonnées : corpus, acquisition et didactique, Rennes : Presses Universitaires de Rennes, 139-158.

CATOIRE Pascale (2017), Entraîner à la compréhension orale en anglais avec l'outil numérique : Les apports du baladeur et des stratégies (thèse de doctorat), Université Paris Descartes, Paris, <https:// hal.archives-ouvertes.fr/tel-01538183> (27 février 2019).

ConSEIL De L’EURope (2001), CECRL (Cadre européen Commun de Référence pour les Langues), Strasbourg : Didier.

CORNAIRE Claudette \& GERMAIN Claude (1998), La Compréhension orale, Paris : CLE international. Chartrand Suzanne-G., EMERY-BRunEAu Judith \& SenEChal Kathleen avec la collaboration de RIVERIN Pascal (2015), Caractéristiques de 50 genres pour développer les compétences langagières en français, Québec : Didactica.

CHARTRAND Suzanne-G. (2016), « Les genres du discours : point nodal de la discipline français », G. Cordeiro \& D. Vrydaghs (dir.), Statut des genres en didactique du français, Namur : Presses universitaires de Namur, 53-81.

D’HAINAUT Louis (1986), L'interdisciplinarité dans l'enseignement général, Paris : Unesco, Division des sciences de l'éducation, des contenus et des méthodes.

De PIETRo Jean-François, MÜLler Catherine \& PicENoni Mathias (2017), « Chansons, musique \& langues : introduction ", Babylonia, 3, 6-9.

De PIETRo Jean-François, Fisher Carole \& GAGNon Roxanne (2017), L'oral aujourd'hui : perspectives didactiques (9), Namur : Presses universitaires de Namur.

DolZ Joaquim \& SCHNEUWLY Bernard (1998), Pour un enseignement de l'oral ; Initiation aux genres formels à l'école, Paris : ESF éditeur.

Dolz Joaquim \& GAGNON Roxane (2008), « Le genre du texte, un outil didactique pour développer le langage oral et écrit », Pratiques, 179-198, <https://journals.openedition.org/pratiques/1159> (27 février 2019).

FIELD John (2008), Listening in the language classroom, Cambridge : Cambridge University Press.

FIELD John (2019), Rethinking the Second Language Listening Test - From Theory to Practice, Sheffield : Equinox. British Council Monographs on Modern Language Testing.

GOMBERT Jean-Émile (1990), Le développement métalinguistique, Paris : Presses universitaires de France. 
HiLToN Heather (2000), « La Didactique de la compréhension aurale : une approche stratégique », Annales de l'Université de Savoie, 28, 23-39.

Koszul Bertrand \& PetitgiraRd Jean-Yves (2014), « COCA "Compréhension orale : conception et assistance" ", LEND - Lingua E Nuova Didattica, 1, 36-40.

MASPERI Monica (2011), « Innovalangues : Innovation et transformation des pratiques de l'enseignement-apprentissage des langues dans l'enseignement supérieur », en ligne sur HAL : $<$ https://hal.archives-ouvertes.fr/hal-02004250> (27 février 2019).

MASPERI Monica \& QUINTIN Jean-Jacques (2014a), « L'innovation selon Innovalangues », LEND Lingua E Nuova Didattica, 1, 6-14.

MASPERI Monica \& QUINTIN Jean-Jacques (2014b), « Enseigner à l'université en France, à l'ère du numérique : l'apport de dispositifs innovants dans la formation en langues », C. Cervini \& A. Valdivieso (dir.), Dispositivi formativi e modalità ibride per l'apprendimento linguistico, Bologna : Edizioni Clueb, 61-80.

PICAVET Francis \& FRost Dan (2014), « Le lot THEMPPO dans le projet Innovalangues : rechercheaction en prosodie et en production orale », LEND - Lingua E Nuova Didattica, 1, 29-35.

PlANE Sylvie \& SCHNEuwly Bernard (2000), « Regards sur les outils de l'enseignement du français : un premier repérage ", Repères, 22, 3-17.

RASTIER François (2011), La mesure et le grain : sémantique de corpus, Paris : Honoré Champion.

SCHNEUWLY Bernard (1995), « Apprendre à écrire. Une approche socio-historique », J.-Y. Boyer, J.P. Dionne \& P. Raymond (dir.), La production des textes, Montréal : Les Éditions Logiques, 73-100.

SCHNEuwly Bernard \& Dolz Joaquim (2009), Des objets enseignés en classe de français - Le travail de l'enseignant sur la rédaction de textes argumentatifs et sur la subordonnée relative, Rennes : Presses Universitaires de Rennes.

SCHNEUWLy Bernard \& AeBy DaGHE Sandrine (2016), « Genres scolaires et genres scolarisés en écriture et pratiques sociales de référence. Le cas de l'école primaire en Suisse romande (1830-2010)», Recherches, 65, 163-175.

THEVENAZ-CHRISTEN Thérèse \& CANELAS-TREVISI Sandra (2018), « L'écriture et la lecture littéraire : une relation qui se transforme », C. Ronveaux \& B. Schneuwly (dir.), Lire des textes réputés littéraires : disciplination et sédimentation. Enquête au fil des degrés scolaires en Suisse romande, 13, Bruxelles : Peter Lang, Théocrit', 305-324.

\section{NOTES}

1. Porté par l'Université Grenoble Alpes, financé par le MESRI et supervisé par l'ANR, Innovalangues (ANR-11-IDFI-0024, 2012-2019) se donne pour but d'impulser et d'accompagner la transformation des pratiques de formation en langues auprès des établissements de l'Enseignement Supérieur, en France et au-delà.

2. L'encadrement scientifique de cette action du projet revient à Sandra Canelas-Trevisi. L'ancrage dans le GRAFE (Université de Psychologie et des Sciences de l'Éducation de Genève), assuré par son intermédiaire (Canelas-Trevisi, 2009a, 2009b, 2015, 2018), a alimenté l'essentiel de nos réflexions et façonné la charpente de l'approche que nous présentons.

3. Pour un éclairage historique de la notion, limpide et condensé, cf. Dolz \& Gagnon, 2008. 
4. La notion de type de discours, telle que la conçoit Bronckart, est ancrée dans un modèle psychosocial de l'activité langagière : l'énonciateur opère un calcul pour sélectionner dans ses connaissances les formes d'organisation discursive les mieux adaptées au but énonciatif qu'il se donne et à sa représentation du destinataire. Chez Adam, la notion de séquentialité désigne un segment de texte ayant des propriétés particulières, analysées selon les principes de la linguistique textuelle. Les recherches de Adam ne sont toutefois ni en ouverte contradiction ni incompatibles avec celles de Bronckart. Aussi, notre reconfiguration didactique s'appuie-t-elle sur des principes supposés universels et sur des démarches d'analyse des données mise en place par la linguistique textuelle.

5. Si les ressources sélectionnées ne sont pas sous licence libre, une procédure de demande de droits d'exploitation pédagogique s'enclenche. La didactisation de la ressource ne peut commencer qu'à partir du moment où l'Institution s'est acquittée de ces droits.

6. Rappelons à titre d'exemple la structuration des séquences par "accent de groupe » qui distingue le français des autres langues romanes ou de l'anglais (caractérisées par un «accent lexical ») et la fonction démarcative de l'accent fixe en français vs fonction distinctive pour les langues à accent libre.

7. Cette application a été conçue, développée et gracieusement mise à disposition du projet par Jean-Jacques Quintin (crea-tice.org).

8. J.-J. Quintin, communication orale, 2017.

9. Par le terme «capacité » nous nous référons à l'opération cognitive (D'Hainaut, 1986) mobilisée dans le traitement du texte.

10. Le visionnage de la vidéo en entier, assorti de la transcription des extraits didactisés, est laissé à la discrétion de l'étudiant, une fois l'exploration de l'unité terminée.

11. À titre d'exemple, nous citerons quelques consignes portant explicitement sur l'extraction d'éléments relevant du type de discours : « attribuer à chaque segment sa fonction explicative »; « identifier les éléments explicatifs qui émergent dans le discours»; « identifier une digression»; " comprendre de quelle manière s'articule le discours explicatif ».

12. La capacité et les consignes sont oralisées en langue cible.

\section{RÉSUMÉS}

Cette contribution s'inscrit dans le cadre d'une recherche en didactique initiée au sein de l'IDEFI Innovalangues (ANR-11-IDFI-0024, Masperi, 2011), portant sur la conception de parcours numériques de formation en langues. Ces réalisations didactiques s'élaborent autour de séquences vidéo sélectionnées sur la base de valeurs culturelles qui les désignent comme étant représentatives de la langue-culture cible. Dans ce contexte, la notion de "genre de texte » fait figure de concept-pivot autour duquel s'échafaude une approche didactique fortement adossée à la recherche fondamentale (en L1, mais quasiment inexplorée en L2) et qui place la langue orale au centre de ses préoccupations.

Nous allons ici questionner d'une part ce parti-pris didactique qui se positionne, sous certains aspects, à contre-courant des pratiques formatives en L2 des dernières décennies. D'autre part nous interrogerons cet objet difficile d'enseignement-apprentissage qu'est l'« oral ", dont la complexité apparaît d'autant plus manifeste que l'on tente, comme nous le proposons, de l'appréhender sans la médiation de l'écrit. 
This paper is part of the researches initiated in the context of the IDEFI Innovalangues project (ANR-11-IDFI-0024, Masperi, 2011), based on the development of e-learning paths in foreign languages. These didactic implementations are conceived around video excerpts, selected on the basis of cultural values that designate them as representatives of the target language. In this context, the notion of "text genre" represents the pivotal idea at the centre of a didactic approach that strongly relies on fundamental research (in L1, but almost unexplored in L2), placing the oral language at the centre of its concern. In this paper we will discuss firstly one part of this didactic commitment that places itself, in some aspects, against the current of the L2 teaching practices of the last decades. Secondly, we will discuss the "oral language" as a teaching object, whose complexity appears to be even more evident as we attempt, as proposed here, to learn it without the mediation of the written language.

INDEX

Mots-clés : texte, genre de texte, type de discours, enseignement-apprentissage L1 et L2, compréhension de l'oral, parcours numériques

Keywords : text, text genre, type of discourse, learning-teaching L1 and L2, oral comprehension, e-learning paths

\section{AUTEURS}

\section{MONICA MASPERI}

Université Grenoble Alpes, LIDILEM

TRISCIA BIAGIOTTI

Université Grenoble Alpes, LIDILEM

GIADA QUARANTA

Université Grenoble Alpes, LIDILEM

MARTINA BARLETTA

Université Grenoble Alpes, LIDILEM 